УДК 621.791.052.8:622.692

ПОВЫШЕНИЕ БЕЗОПАСНОСТИ ПРИ ЭКСПЛУАТАЦИИ УЗЛОВ

ОТВЕТВЛЕНИЯ ТРУБОПРОВОДОВ С УКРЕПЛЯЮЩЕЙ

НАКЛАДНОЙ КОНСТРУКЦИЕЙ ИЗ ПОЛИКАРБАМИДА

\title{
SAFETY IMPROVEMENT UNDER OPERATION OF PIPELINE ANTIFICATION UNITS WITH A STRENGTHENING FIXED POLYUREA CONSTRUCTION
}

\author{
Д.Н. Яковлева, А.В. Исламова
}

Уфимский государственный нефтяной технический университет, Уфа, Российская Федерация

\author{
Darya N. Yakovleva, Alena V. Islamova \\ Ufa State Petroleum Technological University, \\ Ufa, Russian Federation \\ e-mail: daria.yakovleva96@gmail.com
}

Аннотация. Поиск техн ических решений, направленных на повышение работоспособности узлов ответвления трубопровода и их безопасную эксплуатацию, является основной задачей данной работы. Проведен анализ состояния конструкций по обеспечению надежности и безаварийной работы опасных участков трубопровода.

Магистральный трубопровод перекачивает агрессивные среды. Характер нагружения трубопровода влияет на дефекты сборки и приварки накладных колец, самыми опасными из которых являются трещины.

В данной работе предлагается вместо укрепления накладным кольцом использование накладной конструкции из поликарбамида. Накладное кольцо - это плоский элемент, укрепляющий отверстие врезки. Накладная конструкция из поликарбамида представляет собой тело вращения, 
получаемое путем формирования нескольких слоев данного вещества. Поликарбамид (полимочевина) - современное изоляционное покрытие, обладающее целым рядом свойств, которые смогли сделать этот материал самым перспективным среди сегодняшних эластомеров, например быстрое отверждение, устойчивость к воздействию влаги, химических веществ и высоких температур, сопротивляемость на разрыв и растяжение. Поликарбамид широко используется в качестве покрытия стенок резервуаров, подводной части судов.

Использование данного материала исключает дополнительный сварной шов на узле для приварки накладного кольца и остаточные сварочные напряжения, вызванные нагрузками, при которых работает магистральный трубопровод, и являющиеся причиной распространения дефектов на основной трубе соединения с ответвлением. Помимо вышеперечисленных свойств поликарбамид характеризуется большим сроком эксплуатации. Одним из достоинств также является быстрота нанесения поликарбамида, благодаря чему сокращается время проведения работ.

Моделирование напряженно-деформированного состояния узла ответвления с разными геометрическими исполнениями конструкции из поликарбамида показало изменение концентрации напряжений в узле врезки. Определены оптимальные размеры конструкции, обеспечивающие прочность и герметичность узла в рабочих условиях. Геометрия конструкции из поликарбамида определена на основе модели, на которой значения эквивалентных напряжений не превысили допускаемые напряжения для материала узла ответвления. Таким образом, отсутствие сварного шва и прочностные свойства конструкции из поликарбомида позволяют применять данное техническое решение на узлах ответвления магистральных трубопроводов.

Abstract. The search for technical solutions aimed at improving the performance of pipeline branch nodes and their safe operation is the main objective of this work. The condition of the existing structures was analyzed to 
ensure the reliability and trouble-free operation of dangerous sections of the pipeline. The main pipeline pumps aggressive media. The nature of the loading of the pipeline affects defects in the assembly and welding of overhead rings, the most dangerous of which are cracks.

In this paper, it is proposed instead of strengthening the overhead ring using a polyurea patch structure. The overlay ring is a flat element that strengthens the tie-in hole. The polyurea patch construction is a rotational body obtained by forming several layers of a given substance. Polyurea is a modern insulating coating with a number of properties that could make this material the most promising among today's elastomers, such as rapid curing, resistance to moisture, chemicals and high temperatures, resistance to tearing and stretching. Polyurea is widely used as a coating for the walls of tanks, underwater vessels.

The use of this material eliminates the additional weld seam on the site for welding the overlay ring and the residual welding stresses that, due to the loads at which the main pipeline operates, cause defects to spread on the main pipe of the junction with a branch. In addition to the above properties, polyurea is characterized by a long service life. One of the advantages is also the speed of application of polyurea, thereby reducing the time of work.

Modeling of the stress-strain state of a branch node with different geometrical designs of a polyurea structure showed a change in stress concentration in the tie-in unit. The optimal dimensions of the structure are determined to ensure the strength and tightness of the assembly under operating conditions. The geometry of the polyurea structure is determined on the basis of the model in which the equivalent stresses values did not exceed the permissible stresses for the branch node material. Thus, the absence of a welded seam and the strength properties of the polyurea structure make it possible to apply this technical solution at the branch nodes of main pipelines. 
Ключевые слова: трубопровод; трубопроводная система; узел ответвления; сосуды давления; накладное кольцо; поликарбамид

Key words: pipeline; pipeline system; branch node; pressure vessel; ring; polyurea

Технологические трубопроводы имеют большое количество узлов ответвлений (врезок). В случае если в месте врезки ослабление основного трубопровода не компенсируется запасом прочности соединения, то укрепление данных узлов в соответствии с ГОСТ 32569-2013 [1] является необходимой процедурой, поскольку зона врезки является концентратором напряжений из-за уменьшения площади поперечного сечения участка трубопровода.

Рассматривая сосуды, работающие под избыточным внутренним давлением, можно выявить схожий с трубопроводной системой участок отверстие под штуцер. Около 40 \% разрушений сосудов, работающих под давлением, приходится именно на данный участок [2].

Для укрепления опасного участка трубопровода его усиливают специальными накладными конструкциями различных типов:

- плоским накладным кольцом;

- воротником;

- плоским кольцом, охватывающим всю трубу;

- заплечиками;

- кольцом в виде воротника;

- косынками и ребром.

Однако наиболее распространен способ укрепления накладным кольцом в виду того, что теоретически он имеет лучшие прочностные свойства, но фактически в связи со сложностью операций пригонки, сборки и приварки накладного кольца формируются следующие характерные дефекты: 
- трещины;

- укорочение горизонтального катета углового сварного шва «труба кольцо», что стало одной из причин разрушения нефтепровода [3];

- зазоры между усиливающей накладкой и корпусом трубопровода [2];

- дефекты сварного шва;

- эксплуатационные дефекты [3-5].

Bce это ведет к появлению области повышенной концентрации напряжений в узлах врезки и, как следствие, к дальнейшему разрушению.

Анализ отчетов об авариях, например, на нефтебазе Шесхарис [6], показал, что укрепление накладным кольцом, действительно, не обеспечивает необходимую прочность и герметичность узла.

Целью исследования является разработка технических решений для устранения вышеописанной проблемы.

Учитывая сложность технологии изготовления и монтажа накладных колец, предлагается использование укрепляющего элемента из поликарбамида, тем самым исключив необходимость в сварных соединениях и, как следствие, зону термического влияния, а также дефекты сварных швов (рисунок 1).

Поликарбамид обладает следующими основными свойствами:

- твёрдость по Шору (А) при сохранении эластичности - 83-98;

- сопротивление к ударам при минус $20{ }^{\circ} \mathrm{C}-50-100$ кДж/м²

- срок службы покрытия из поликарбамида - до 50 лет в зависимости от качества поликарбамида и условий нанесения;

- высокая адгезия к поверхности (металл, бетон) - 15 МПа [7];

- максимальная рабочая температура от минус 100 до $+250{ }^{\circ} \mathrm{C}$. 


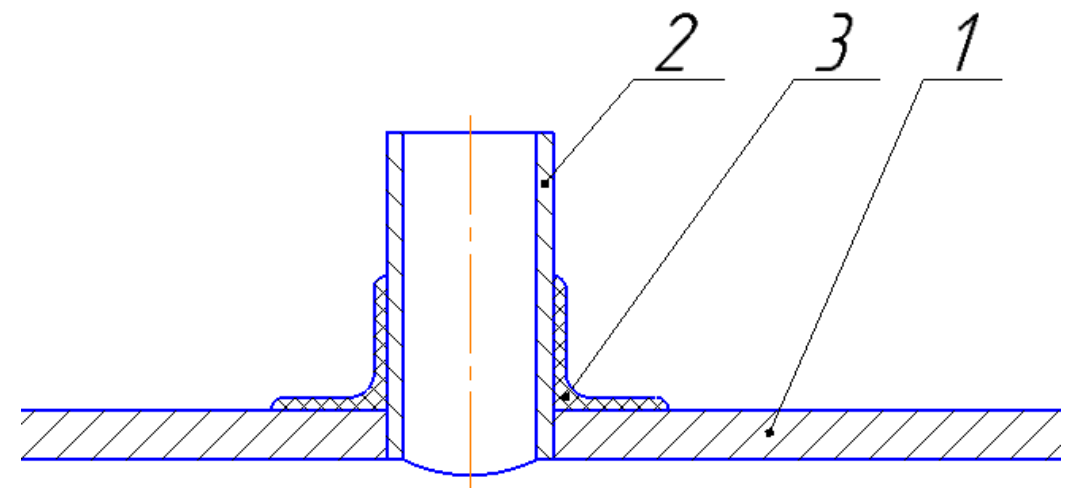

1 - основная труба; 2 - ответвление трубы;

3 - усиливающая накладка из поликарбамида

Рисунок 1. Схема сварного ответвления с использованием укрепляющего элемента из поликарбамида

При использовании укрепляющего элемента из поликарбамида его нанесение исключает зазор между накладкой и корпусом.

С целью определения и сравнения напряженно-деформированного состояния укрепления модели узла ответвления металлическим кольцом и различными конструктивными исполнениями накладок из поликарбамида были проведены численные исследование на моделях в программе ANSYS WORCKBENCH в модуле Static Structural.

Модель представляла собой цилиндрическую трубу с врезанным патрубком, нагруженная внутренним избыточным давлением. Расчетные данные модели приведены в таблице 1.

Таблица 1. Расчетные данные модели

\begin{tabular}{|l|c|c|}
\hline \multicolumn{1}{|c|}{ Показатель } & Единица & Значение \\
\hline Наружный диаметр основного трубопровода & мм & 820 \\
\hline Толщина стенки основного трубопровода & мм & 12 \\
\hline Рабочее давление & МПа & 1,6 \\
\hline Температура & ${ }^{\circ} \mathrm{C}$ & 20 \\
\hline Наружный диаметр трубопровода-патрубка & мм & 219 \\
\hline Толщина стенки трубопровода-патрубка & мм & 4 \\
\hline
\end{tabular}


Место врезки и укрепления отверстия усиливалось покрытием из поликарбамида путем сборки основной трубы с моделью накладной конструкции (рисунок 2). Остаются неопределенными размеры конструкции для соединения с ответвлением, так как время отверждения поликарбамида 20 c, и необходимо точно задать их для нанесения непосредственно на трубопроводе.

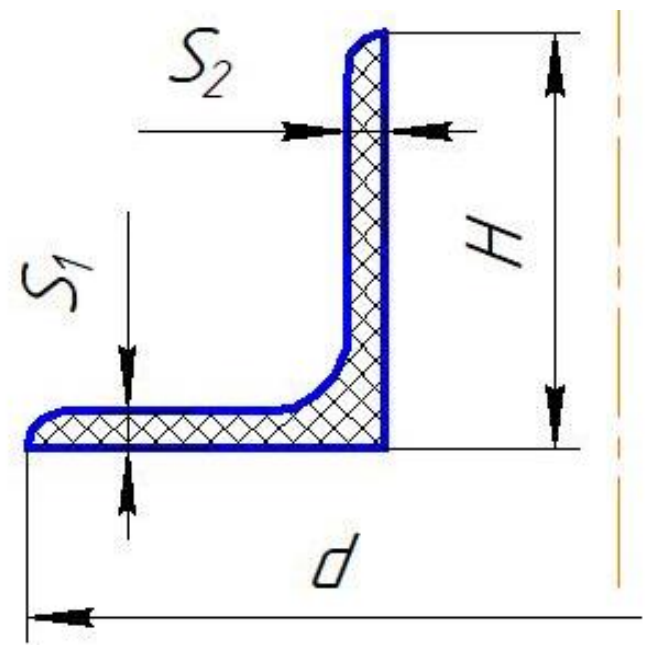

Рисунок 2. Основные размеры покрытия

Для проведения выбора оптимального варианта были рассмотрены модели с различными размерами укрепляющего элемента. Размеры укрепляющего элемента представлены в таблице 2. Размер Н задается в пределах 30 \% от диаметра накладной конструкции.

Таблица 2. Размеры укрепляющего элемента

\begin{tabular}{|l|c|c|c|c|}
\hline & $\mathrm{d}$, мм & $\mathrm{H}, \mathrm{Mм}$ & $\mathrm{S}_{1}, \mathrm{MM}$ & $\mathrm{S}_{2}, \mathrm{Mм}$ \\
\hline Модель 1 & 350 & 100 & 12 & 4 \\
\hline Модель 2 & 400 & 150 & 12 & 4 \\
\hline Модель 3 & 450 & 200 & 12 & 4 \\
\hline
\end{tabular}

Основные размеры расчетной модели показаны на рисунке 3. 


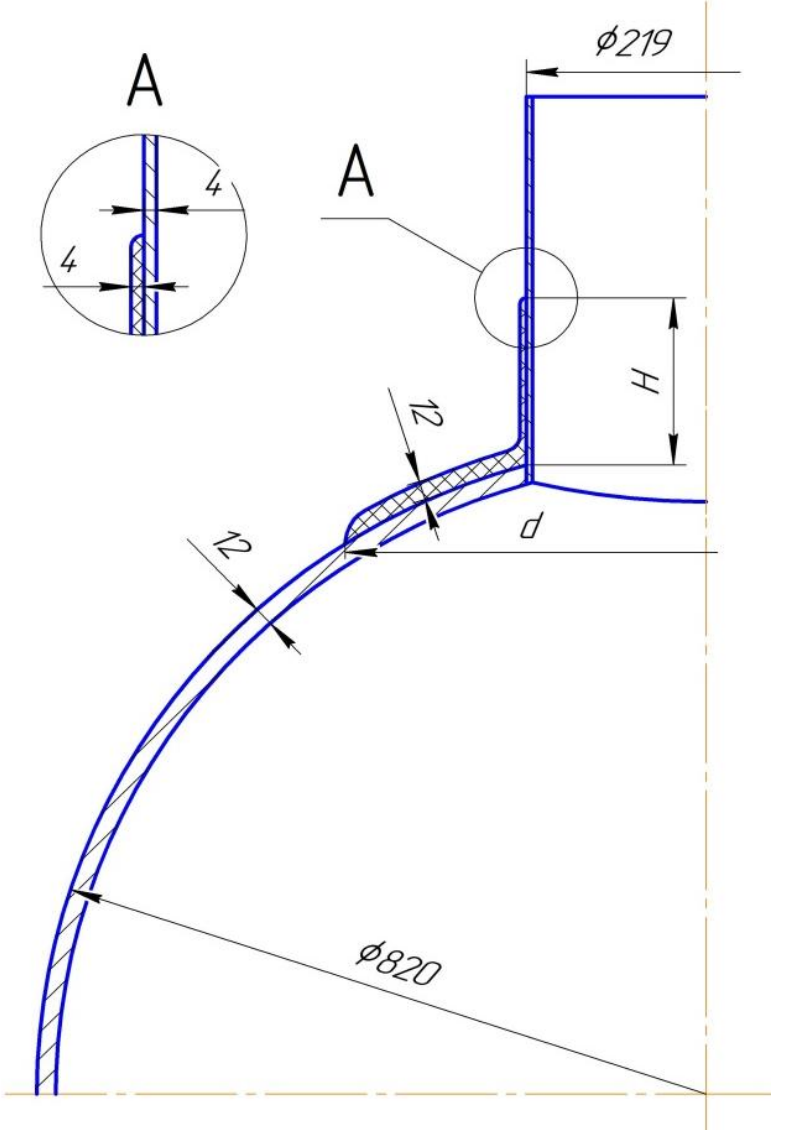

Рисунок 3. Основные размеры расчетной модели

После импорта моделей из Компас 3D в ANSYS WB задавали свойства материалов:

- основная труба и патрубок 09Г2С (предел прочности $\sigma_{\mathrm{B}}=450 \mathrm{MПа}$ ГОСТ 10705-80 [8]);

- покрытие - поликарбамид (предел прочности на разрыв 60 МПа, плотность 1101,8 кг/м³ , твёрдость 683 НВ).

Для наиболее точных результатов необходимо построить регулярную сетку с минимально возможным размером элементов. Общий вид построенной модели и сетка конечных элементов показаны на рисунке 4.

Исследуемая модель рассматривалась в поперечном сечении. Для построения графиков эквивалентных напряжений были выбраны точки на основной трубе вдоль поперечного сечения (рисунок 5).

График изменения эквивалентных напряжений наружной поверхности трубы по поперечному сечению представлен на рисунке 6. 


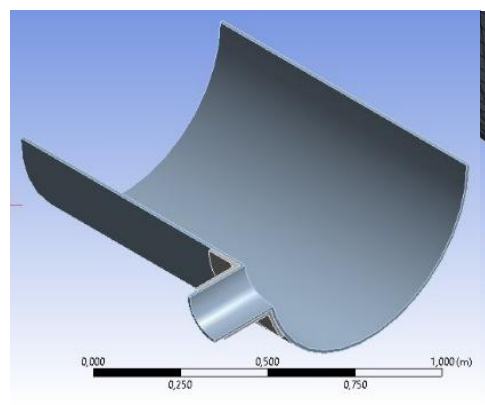

a)

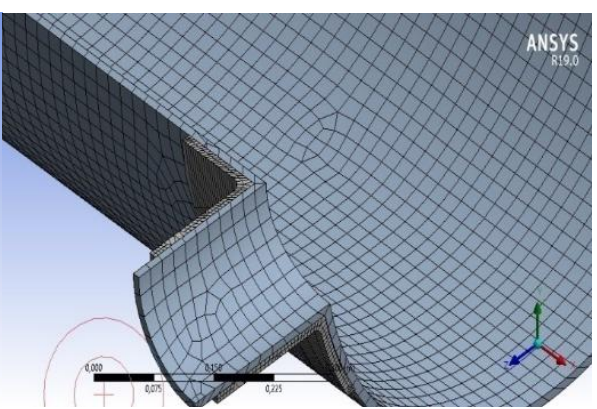

б)

Рисунок 4. Общий вид построенной модели (а) и сетка конечных элементов (б)

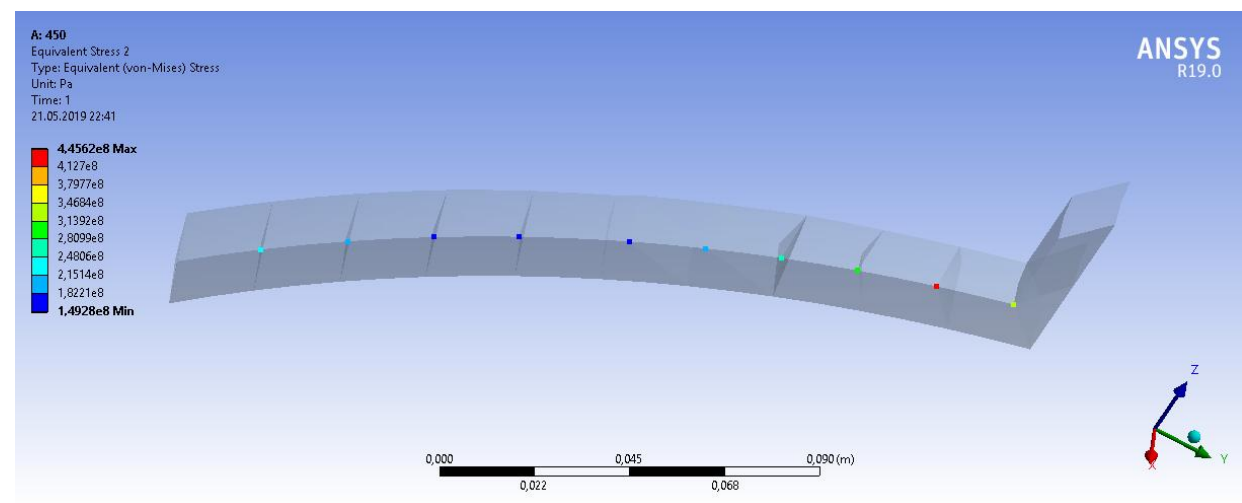

Рисунок 5. Точечное распределение эквивалентных напряжений

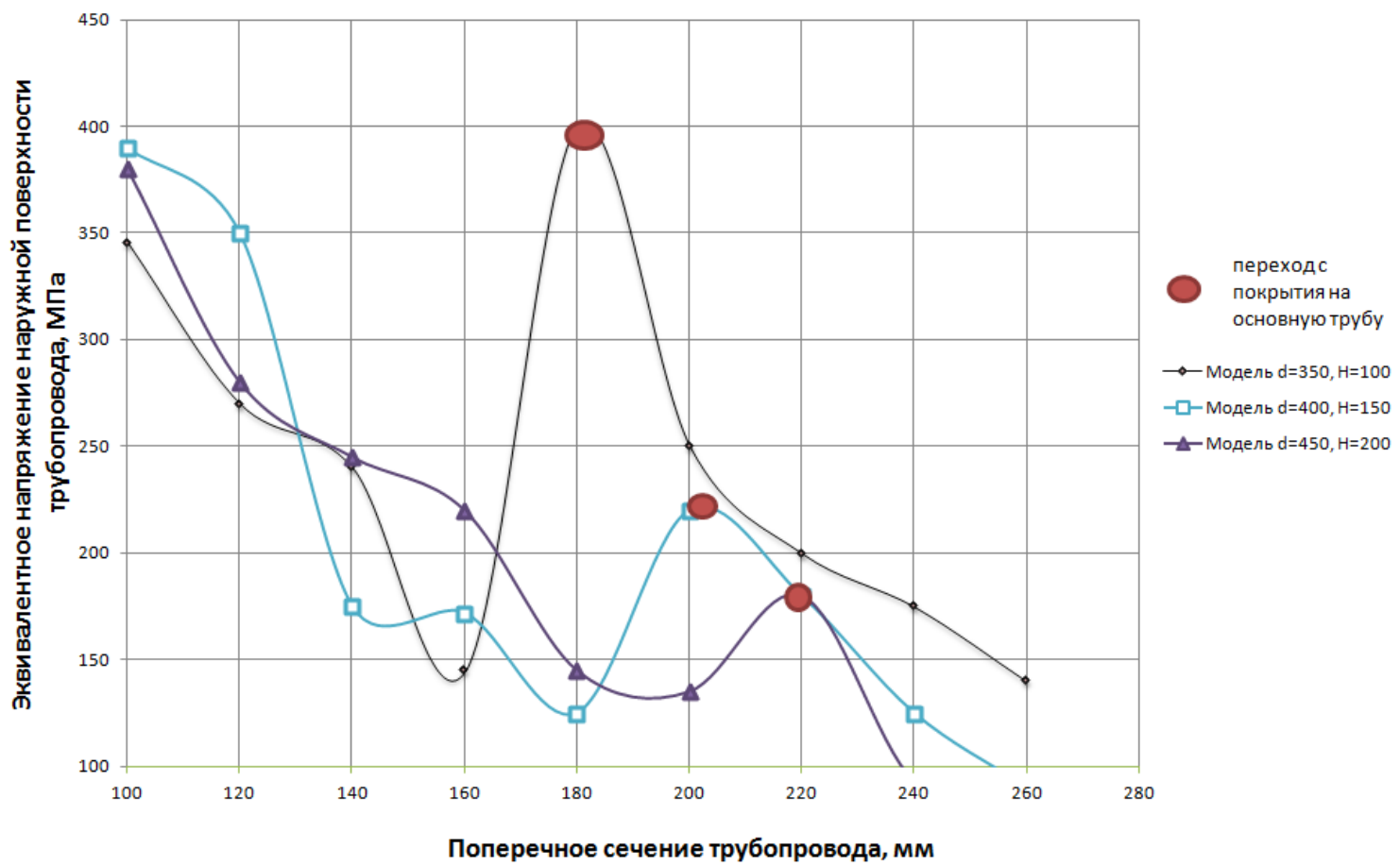

Рисунок 6. График изменения эквивалентных напряжений наружной поверхности трубы вдоль поперечного сечения трубы 
Переход основной трубы с покрытием на основную трубу без покрытия является аналогом сварного шва для укрепления накладным кольцом.

Как показывают результаты моделирования, при использовании укрепляющих элементов диаметром 400 и 450 мм эквивалентные напряжения в основной трубе и патрубке не превышают допускаемые напряжения материала. Модель $(\mathrm{d}=350$ мм; $\mathrm{H}=100$ мм) с максимальным напряжением 400 МПа считается критической.

Следовательно, в рамках данного исследования оптимальный размер покрытия:

- диаметр d = 400 мм;

- высота Н = 150 мм.

\section{Выводы}

1. Предложен способ укрепления узла из поликарбамида вместо традиционного укрепления приварным укрепляющим кольцом. Альтернативный способ позволяет исключить необходимость в сварных соединениях и, как следствие, зону термического влияния, а также дефекты сварных швов.

2. На основании проведенного численного моделирования на примере модели «трубопровод - патрубок» было установлено, что укрепление накладными кольцами можно заменить на укрепляющий элемент из поликарбамида.

Можно предположить, что размеры покрытия следующие:

- диаметр наносимого покрытия системой горячего безвоздушного распыления на основной трубе $\mathrm{D}_{\text {покр }} \geq 2 \mathrm{~d}_{\text {пат }}$;

- толщина покрытия на патрубке и основной трубе равна толщине стенки основной трубы, что обеспечивается путем нанесения нескольких слоев поликарбамида (толщина 1 слоя = 4 мм);

- высота покрытия на патрубке равна $\mathrm{H} \geq 0,75 \cdot \mathrm{d}_{\text {пат }}$. 
Для подтверждения вышесказанных предположений необходимо проводить аналогичные исследования с другими типоразмерами модели.

\section{Список используемых источников}

1. ГОСТ 32569-2013. Трубопроводы технологические стальные. Требования к устройству и эксплуатации на взрывопожароопасных и химически опасных производствах. М.: Межгосуд. совет по стандартизации, метрологии и сертификации, 2015. 131 с.

2. Аписов И.В., Четверткова О.В., Каретников Д.В., Ибрагимов И.Г., Закиров Т.Э. Анализ напряженного состояния укрепленного накладным кольцом штуцерного узла с учетом дефектов сборки // Электронный научный журнал «Нефтегазовое дело». 2014. № 5. С. 223-237. URL: http://ogbus.ru/files/ogbus/issues/5_2014/ogbus_5_2014_p223237_ApisovIV_ru.pdf.

3. Гумеров А.К., Шмаков А.К., Хайрутдинов Ф.Ш. Механизмы разрушения магистральных трубопроводов с приварными элементами // Электронный научный журнал «Нефтегазовое дело». 2007. №1. С. 1-5. URL: http://ogbus.ru/files/ogbus/authors/GumerovAK/GumerovAK_1.pdf.

4. Хасанов Р.Р., Султанмагомедов С.М. Напряженно-деформированное состояние и обеспечение надежности тройников подземных трубопроводов. Уфа: Изд-во УГНТУ, 2013. 100 с.

5. Файрушин А.М., Зарипов М.З., Каретников Д.В, Яковлева Д.Н., Исламова А.В. Разработка рекомендаций по применению локально укрепленных штуцерных узлов с ребрами жесткости // Электронный научный журнал «Нефтегазовое дело». 2017. № 6. C. 76-95. URL: http://ogbus.ru/files/ogbus/issues/6_2017/ogbus_6_2017_p7695_FairushinAM_ru.pdf. 
6. Гумеров К.М., Багманов P.P. Человеческий фактор при конструировании и эксплуатации сложных узлов нефтепроводов // Современные фундаментальные и прикладные исследования. 2016. № 4. C. $72-80$.

7. Пивоваров В.Ю., Исламова А.В. Влияние покрытия из полимочевины на физико-механические свойства стали и бетона // Сетевое издание «Нефтегазовое дело». 2018. № 3. С. 29-42. URL: http://ogbus.ru/files/ogbus/issues/3_2018/ogbus_3_2018_p29-42.pdf.

8. ГОСТ 10705-80. Трубы стальные электросварные. Технические условия. М.: Министерство черной металлургии СССР, 1982. 10 с.

\section{References}

1. GOST 32569-2013. Truboprovody tekhnologicheskie stal'nye. Trebovaniya $k$ ustroistvu $i$ ekspluatatsii na vzryvopozharoopasnykh $i$ khimicheski opasnykh proizvodstvakh [State Standard 32569-2013. Industrial Steel Pipelines. Requirements for Design and Operation in Explosive and Chemically Dangerous Industries]. Moscow, Mezhgosud. sovet po standartizatsii, metrologii i sertifikatsii Publ., 2015. 131 p. [in Russian].

2. Apisov I.V., Chetvertkova O.V., Karetnikov D.V., Ibragimov I.G., Zakirov T.E. Analiz napryazhennogo sostoyaniya ukreplennogo nakladnym kol'tsom shtutsernogo uzla s uchetom defektov sborki [Deflected Mode's Analyze of Vessel's Connecting Pipe Strengthened with Restorative Ring]. Elektronnyi nauchnyi zhurnal «Neftegazovoe delo»-Electronic Scientific Journal «Oil and Gas Business», 2014, No. 5, pp. 223-237. URL: http://ogbus.ru/files/ogbus/issues/5_2014/ogbus_5_2014_p223237_Api-sovIV_ru.pdf. [in Russian]. 
3. Gumerov A.K., Shmakov A.K., Khajrutdinov F.S. Mekhanizmy razrusheniya magistral'nykh truboprovodov $\mathrm{s}$ privarnymi elementami [Destruction Mechanisms of Trunk Pipelines with Welded Elements]. Elektronnyi nauchnyi zhurnal «Neftegazovoe delo»-Electronic Scientific Journal «Oil and Gas Business», 2007, No. 1, pp. 1-5. URL: http://ogbus.ru/files/ogbus/authors/GumerovAK/GumerovAK_1.pdf. [in Russian].

4. Khasanov R.R., Sultanmagomedov S.M. Napryazhennodeformirovannoe sostoyanie i obespechenie nadezhnosti troinikov podzemnykh truboprovodov [Stress-Strain State and Ensuring the Reliability of Tees Underground Pipelines] Ufa, UGNTU Publ., 2013. 100 p. [in Russian].

5. Fajrushin A.M., Zaripov M.Z., Karetnikov D.V, Yakovleva D.N., Islamova A.V. Razrabotka rekomendatsii po primeneniyu lokal'no ukreplennykh shtutsernykh uzlov s rebrami zhestkosti [Development of Recommendations on the Application of Locally Strengthened Vessel's Connecting Pipes with Enforcement Ribs]. Elektronnyi nauchnyi zhurnal «Neftegazovoe delo» - Electronic Scientific Journal «Oil and Gas Business», 2017, No. 6, pp. 76-95. URL: http://ogbus.ru/files/ogbus/issues/6_2017/ogbus_6_2017_p76-95_Fai-rushin AM_ru.pdf. [in Russian].

6. Gumerov K.M. Chelovecheskii faktor pri konstruirovanii i ekspluatatsii slozhnykh uzlov nefteprovodov [Human factor in the design and operation of complex nodes of pipelines] Sovremennye fundamental'nye $i$ prikladnye issledovaniya - Modern Basic and Applied Research. 2016. No. 4. pp. 72-80.

7. Pivovarov V.Yu., Islamova A.V. Vliyanie pokrytiya iz polimocheviny na fiziko-mekhanicheskie svoistva stali i betona [The Coating Effect of Polyurea on the Physico-Mechanical Properties of Steel and Concrete] Setevoe izdanie «Neftegazovoe delo» - Online Edition «Oil and Gas Business», 2018, No. 3, pp. 29-42. URL: http://ogbus.ru/files/ogbus/issues/3_2018/ogbus_3_2018 p29-42.pdf. [in Russian]. 
8. GOST 10705-80. Truby stal'nye elektrosvarnye. Tekhnicheskie usloviya [State Standard 10705-80. Electrically Welded Steel Tubes. Specifications]. Moscow, Ministerstvo chernoi metallurgii SSSR Publ., 1982. 10 p. [in Russian].

\section{Сведения об авторах}

\section{About the authors}

Яковлева Дарья Николаевна, магистрант кафедры «Технология нефтяного аппаратостроения», УГНТУ, г. Уфа, Российская Федерация

Darya N. Yakovleva, Undergraduate Student of Technology of Petroleum Apparatus Construction Department, USPTU, Ufa, Russian Federation

e-mail: daria.yakovleva96@gmail.com

Исламова Алена Вячеславовна, студент кафедры «Технологические машины и оборудование», УГНТУ, г. Уфа, Российская Федерация

Alena V. Islamova, Student of Technological Machines and Equipment Department, USPTU, Ufa, Russian Federation

e-mail: alena.islamova.1997@mail.ru 